\title{
Rechazo parental en homosexuales de una unidad de medicina familiar
}

\author{
Rejeição parental em homossexuais de uma unidade de medicina familiar

\section{Parental rejection of homosexuals in a family primary health care unit}

Donovan Casas Patiño. Centro Universitario-Amecameca de la Universidad Autónoma del Estado de México (UAEM). Amecameca, Estado de México, México. capo730211@yahoo.es (Autor correspondiente)

Alejandra Rodríguez Torres. Unidad de Medicina Familiar 195 del Instituto Mexicano del Seguro Social (IMSS). Chalco, Estado de México, México. aledefra2001@yahoo.com.mx

Camila Pereira Abagaro. Unidad Académica Profesional-Nezahualcóyotl de la Universidad Autónoma del Estado de México (UAEM). Nezahualcóyotl, Estado de México, México. camila.abagaro@gmail.com

\section{Resumen}

Objetivo: Conocer la frecuencia de rechazo parental en pacientes homosexuales de una unidad de medicina familiar. Métodos: estudio de tipo descriptivo donde se aplicó el instrumento de Rechazo Familiar de Lozano-Díaz (2010) a 39 padres de familia de homosexuales adscritos a la Unidad de Medicina Familiar n 195 de Chalco, México. La muestra no probabilística y por conveniencia se obtuvo en la consulta de medicina familiar, con ayuda de trabajadores sociales, concertando citas con los padres de pacientes reconocidos como homosexuales. Resultados: 1) Hubo una mayor actitud negativa hacia la homosexualidad por la rama paterna; 2) Hubo un alto sentimiento de deshonra familiar por tener en la familia un hijo o hija homosexual; 3) Se consideró altamente desagradable la preferencia sexual hacia el mismo sexo; 4) № se aceptan los lazos matrimoniales entre parejas del mismo sexo. Conclusiones: Se puede afirmar que el rechazo parental al hijo/hija homosexual fue considerablemente alto en el grupo investigado. Cabe mencionar que estos pacientes deben ser abordados no sólo individualmente sino en su entorno familiar. La búsqueda de alternativas no biomédicas puede ayudar a la aceptación de la expresión homosexual, disminuyendo la discriminación en el ambiente familiar, y, por ende, en el institucional.

\section{Resumo}

Objetivo: Conhecer a frequência de rejeição parental em pacientes homossexuais de uma unidade de medicina familiar. Métodos: Estudo do tipo descritivo, no qual foi aplicada a escala de 'Rejeição familiar' de Lozano-Díaz (2010) a 39 pais de família de pacientes homossexuais adscritos à Unidade de Medicina Familiar nº 195 de Chalco, México. A amostra não probabilística e por conveniência foi obtida na consulta de Medicina Familiar, com a ajuda do serviço social, marcando consultas com os pais de pacientes reconhecidos como homossexuais. Resultados: 1) Houve uma maior atitude negativa frente à homossexualidade pelo lado paterno; 2) Houve um alto sentimento de desonra familiar por ter na família um filho ou uma filha homossexual; 3) Considerou-se altamente desagradável a preferência sexual pelo mesmo sexo; 4) Não se aceitam os laços matrimoniais entre casais do mesmo sexo. Conclusões: Pode-se afirmar que a rejeição parental em relação a filho/filha homossexual foi consideravelmente alta no grupo pesquisado. Cabe mencionar que estes pacientes devem ser abordados não somente individualmente, mas também em seu entorno familiar. A busca de alternativas não biomédicas pode auxiliar uma aceitação da expressão homossexual, diminuindo a discriminação no ambiente familiar e, consequentemente, no ambiente institucional.

\section{Abstract}

Objective: To know the frequency of parental rejection in homosexual patients in a family primary health care unit. Methods: A descriptive study carried out by the application of the Family Rejection instrument by Lozano-Díaz (2010) to 39 parents of homosexual patients assigned to the Family Medicine Unit $n^{0} 195$ in Chalco, Mexico. The non-probabilistic convenience sample was obtained in family medicine consultations and appointments with the parents of patients recognized as homosexuals were arranged with the help of social workers. Results: 1) The worst negative attitude towards homosexuality was observed in the fathers; 2) There was a great feeling of family dishonor to have a homosexual son or daughter; 3) It was considered very unpleasant to have sexual preference for the same sex; 4) Marriages were not accepted between same-sex couples. Conclusions: It is possible to state that the parental rejection of homosexuals was considerably high in the group investigated. It is noteworthy that these patients need to be addressed not only individually, but also with their families. The search for non-biomedical alternatives can provide an opportunity for the acceptance of homosexual expression, reducing discrimination within the family unit, and hence in social environments.

Como citar: Casas Patiño D, Rodríguez Torres A, Pereira Abagaro C. Rechazo parental en homosexuales de una unidad de medicina familiar. Rev Bras Med Fam Comunidade. 2014;9(31): 127-32. Disponível em: http://dx.doi.org/10.5712/rbmfc9(31)547

\section{Palabras clave:}

Homosexualidad

Medicina Familiar y Comunitaria Homofobia

Palavras-chave: Homossexualidade Medicina de Família e Comunidade Homofobia

Keywords:

Homosexuality Family Practice Homophobia

\section{Financiación:}

ninguna declarada.

Aprobación ética:

Comité Local Multidisciplinario de Investigación Nezahualcóyotl, del Instituto Mexicano de Seguro Social 2012-02-06.

Conflicto de intereses: ninguno declarado. Recibido el: 19/08/2012. Aceptado el: 29/12/2013. 


\section{Introducción}

A lo largo del tiempo la familia ha sido objeto de múltiples cambios y transformaciones. Su evolución y significación está determinada por el proceso socio-cultural y económico de cada región. ${ }^{1-3}$ Esto ha sido estudiado por sociólogos, psicólogos, antropólogos, abogados y es algo que el médico de atención primaria debe también comprender. ${ }^{4-6} \mathrm{El}$ hecho de que la propia historia familiar ha provocado el desarrollo social de hoy en día, ha sido objeto de reflexión con los alcances y límites de sus primeros estadios, pues se trata de una compleja organización social que ha enfrentado, hasta la fecha, multifacéticos problemas que han permitido la continuidad y supervivencia de la especie humana.-9

La familia determina la adaptabilidad del individuo a la sociedad - por lo que se trata de un entorno social entrópico de reproducción social inevitablemente cargado de historia - y también se puede acotar que el ser humano es un modelo modificable en sus derechos y obligaciones, ${ }^{1,5,10}$ responsable tanto en el ámbito privado como en el público, la cual interesa a la sociedad de cada momento histórico porque es ahí donde se reproduce la actividad de cada sociedad, donde el individuó es formado y adoctrinado con lo que debe ser y con lo que debe hacer. ¿Qué sucede cuando esto no se cumple? Respecto a la preferencia sexual, saber, sin ningún género de dudas, si este individuo es aceptado o rechazado por el núcleo familiar es de suma importancia para la Medicina Familiar (MF) puesto que "la familia" es el objeto de su especialidad, la cual juega el papel más importante dentro del ámbito de esta profesión. La familia y la especialidad en medicina familiar evolucionan al unísono, y, por tanto, se necesitan herramientas de comprensión y análisis de toda la diversidad sexual en las personas que acuden a la atención primaria.

Aunado a lo anterior se puede afirmar que la familia es un objeto complejo, cuya complejidad radica en que sus miembros interactúan en un espacio social articulado con otras estructuras sociales, que teje y construye relaciones horizontales y verticales, directas y alternas con otros espacios de la sociedad. Entender la familia desde el enfoque de la medicina familiar nos ayuda a entender los desiguales cambios de adaptación a que se enfrenta el individuo que busca su adaptación social en un mundo sexualmente diversificado.

El médico de familia debe estar capacitado para orientar, derivar y atender, sin prejuicios científicos o sociales, a las familias que presenten una disfunción familiar originada por diferencias en la preferencia sexual de alguno de sus integrantes. Por este motivo se hace prioritario, en la Atención Primaria de Salud (APS), investigar la actitud de los familiares que tienen en su núcleo a un homosexual, puesto que aquí se puede brindar atención y prevención del daño familiar en cada integrante, y así contener y derivar oportunamente a los servicios de psicología y trabajo social, para una integración familiar derivada de la diferencia en la preferencia sexual. Cabe mencionar que hasta este momento no existe una investigación de esta índole en la APS en México y no se cuenta con datos estadísticos respecto a las personas con diferencias en la preferencia sexual. La significativa evolución de la familia y la apertura sexual en este siglo obliga a los profesionales de la APS y de la medicina familiar a tener una postura de análisis respecto a la preferencia sexual diferente de la que tienen las personas del núcleo familiar, para poder intervenir de manera acertada en favor de la integración familiar.

La homosexualidad es una orientación sexual y se define como la interacción o atracción sexual, emocional, sentimental y afectiva hacia individuos del mismo sexo. Etimológicamente, la palabra homosexual es un híbrido del griego homós (igual) y del adjetivo latino sexualis, lo que sugiere una relación sexual y sentimental entre personas del mismo sexo. El término fue empleado por primera vez en 1869 por Karl-Maria Kertbeny, y el libro Psychopathia Sexualis de Richard Freiherr von KrafftEbing popularizó el concepto, en 1886. La palabra gay es un adjetivo o sustantivo que identifica a las personas homosexuales, sin importar su género. Desde 1973, la comunidad científica internacional considera que la homosexualidad no es una enfermedad, sino una preferencia sexual. ${ }^{11-13}$ Sin embargo el CIE 10 de la Clasificación Internacional de Enfermedades de la Organización Mundial de la Salud (OMS) en su capítulo relativo a los "trastornos mentales y del comportamiento" (F668-número asignado para codificación internacional) continua patologizando a la homosexualidad, reforzando con esto la supremacía, dominancia y legitimidad de las prácticas heterosexuales, y, por otro lado, discriminando y estigmatizando la homosexualidad, 5,14 junto con toda aquella manifestación visible de comportamiento o erotismo que se salga del "círculo mágico" de la sexualidad heterosexual. ${ }^{5}$

En el mismo sentido, la reproducción de valores, códigos y significados del sistema sexo/género que determina las características que debe tener un "verdadero hombre" y una "buena mujer" - a través del modelo patriarcal, androcéntrico y heterosexista - y que continúa con los discursos condenatorios hacia la diversidad sexual, con los que estos individuos se invisibilizan, degradan o asesinan, sin importar su condición de persona y todo esto por el mero hecho de buscar la expresión libre de su sexualidad. Anclar esta investigación en la APS es brindar la oportunidad para que se abran nuevas puertas a este mundo cambiante, donde impere la dignidad humana y la igualdad de derechos tanto individual como colectivamente, donde la medicina familiar aporte elementos de participación con los que entender que la diversidad sexual no es una anormalidad. 


\section{Métodos}

Con este marco teórico se llevó a cabo una investigación de tipo descriptivo, en una Unidad de Medicina Familiar (UMF) en el límite oriental del Estado de México, perteneciente al Instituto Mexicano del Seguro Social (IMSS) ubicada en el municipio de Chalco. Se utilizó el instrumento de Lozano-Díaz ${ }^{15,16}$ por dos razones: a) El instrumento cuenta con alto nivel de confiabilidad $(0,9)$ y validez interna, y; b) El instrumento tiene cuatro factores (indicadores o categorías) que son de suma importancia: 1) Discriminación a la expresión homosexual - se vincula con un menosprecio hacia la homosexualidad, con prejuicios y mitos; 2) Rechazo familiar - describe la conducta asumida en la familia hacia la homosexualidad y hacia los miembros homosexuales de otros grupos familiares; 3) Rechazo social - describe el rechazo basado en normas de importantes instituciones sociales, como la iglesia y la familia; y 4) Rechazo personal - se trata de creencias y conductas provenientes de individuos que no aceptan la expresión homosexual. Para esta investigación es importante subrayar que la categoría "rechazo familiar" cumple con el objetivo planteado en la investigación, por lo cual únicamente implementamos este fragmento, y tiene una escala de calificación que abarca los siguientes niveles: actitud positiva, actitud favorable, actitud indiferente y actitud desfavorable.

Se aplicó este instrumento en su categoría "Rechazo Familiar", con previo consentimiento informado, a 39 padres de familia con hijos homosexuales adscritos a la UMF 195 "Chalco" IMSS. La muestra fue obtenida, de manera no probabilística y por conveniencia, en la consulta de medicina familiar, con ayuda de los trabajadores sociales, en un período de seis meses (de marzo a diciembre del ańo 2011) interrogando a los usuarios respecto a su preferencia sexual. De este grupo se categorizó su preferencia sexual de la siguiente manera: gay, lesbiana y bisexual. Se concertaron citas con los padres y los hijos homosexuales adscritos a la UMF 195 para que, una vez obtenido el permiso por ambas partes, se autorizara al equipo de trabajadores sociales la aplicación del instrumento.

Este estudio fue aprobado por el Comité de Ética en Pesquisa del Comité Local Multidisciplinario de Investigación Nezahualcóyotl, del Instituto Mexicano de Seguro Social, protocolo de investigación número 2012/02/06.

\section{Resultados}

Se estudió a 39 padres y madres de pacientes homosexuales de los cuales $28(71,7 \%)$ eran mujeres y $11(28,3 \%)$ eran hombres. La media de edad para los familiares fue de 43 años con un valor mínimo de 32 años y máximo de 53 años. Respecto a la categorización de los pacientes encontramos 21 gays y 8 lesbianas, la edad media fue de 23 años, con un valor mínimo de 17 años y máximo de 31 años.

El instrumento de Lozano-Díaz mostró las siguientes variables: a) La deshonra para una madre al enterarse de que su hijo/a es homosexual; b) La deshonra para un padre al enterarse de que su hijo/a es homosexual; c) La peor deshonra para una familia es que su hijo/a sea homosexual; d) Yo creo que los homosexuales son desagradables, y e) La idea de matrimonio homosexual la encuentro ridícula (Cuadro 1).

Respecto a "deshonra para un padre o madre si su hijo/a es homosexual", por género, encontramos que los hombres, al igual que las mujeres, obtuvieron una muy alta actitud desfavorable. Respecto a actitud indiferente no existió una diferencia significativa entre los géneros, y respecto a actitud favorable sólo una madre contestó afirmativamente, lo cual podría ser un sesgo. En cuanto a "deshonra familiar por tener un hijo o hija homosexual" encontramos una muy alta actitud desfavorable, al igual que baja puntuación en actitud indiferente, y sólo un familiar respondió favorablemente.

Cuadro 1. Actitud de los padres y madres de hijos homosexuales adscritos a la UMF 195 IMSS, Chalco, México, 2011.

\begin{tabular}{lcccc}
\hline \multicolumn{1}{c}{ Variable } & Actitud Desfavorable & Actitud Indiferente & Actitud Favorable & Actitud Positiva \\
\hline Deshonra para una madre al enterarse que su hijo/a es homosexual & $75 \%$ & $21,4 \%$ & $3,6 \%$ & $0 \%$ \\
Deshonra para un padre al enterarse de que su hijo/a es homosexual & $81,8 \%$ & $18,2 \%$ & $0 \%$ & $0 \%$ \\
La peor deshonra para una familia es que su hijo/a sea homosexual & $84,6 \%$ & $12,8 \%$ & $2,6 \%$ & $0 \%$ \\
Yo creo que los homosexuales son desagradables & $76,9 \%$ & $23,1 \%$ & $0 \%$ & $0 \%$ \\
La idea de matrimonio homosexual la encuentro ridícula & $89,8 \%$ & $7,6 \%$ & $2,6 \%$ & $0 \%$ \\
\hline
\end{tabular}

Origen: Datos obtenidos del concentrado de la categoría Rechazo Familiar. 
Se obtuvieron en la categoría d), "Yo creo que los homosexuales son desagradables", un muy alto grado de actitud desfavorable, un muy bajo grado de actitud indiferente, con nula respuesta tanto en actitud favorable como en actitud positiva.

La proporción de padres y madres que puntuó la categoría e), "idea de matrimonio homosexual como ridícula", demuestra una muy alta actitud desfavorable y muy baja actitud indiferente, con solo un familiar siendo favorable.

\section{Discusión}

En esta investigación observamos que el rechazo de un padre o madre si su hijo/a es homosexual es muy alto, con una actitud desfavorable en ambos géneros. Este condicionante es muy importante puesto que el padre o la madre son los jefes de familia, y de ellos depende la construcción cultural respecto a la homofobia en nuestra sociedad. Los mitos y preceptos iniciales respecto a la homofobia se prepararon para el nuevo individuo dentro de una familia.

Respecto a la variable "deshonra familiar por un hijo/a homosexual", encontramos una muy alta actitud desfavorable al igual que la percepción respecto a la variable "yo creo que los homosexuales son desagradables". La familia construye significados de la cultura, y los toma como propios, reproduciéndolos en los individuos que conforman la familia. En este caso podemos observar que la deshonra y el rechazo se conforman dentro de la familia como violencia simbólica aprendida en el seno familiar y que se reproduce ilimitadamente en lo social: por un lado la familia constituye un significado de gran importancia hacia la homofobia y por otro lado los medios masivos de comunicación lo refuerzan con mofas y señalamientos a aquellos que practican la diversidad sexual.

Un elevado rechazo por parte de la familia hacia la orientación o identidad sexual del hijo, en jóvenes homosexuales y transexuales, multiplica por ocho el riesgo de suicidio, mientras que también incrementa las posibilidades de que el joven consuma drogas y de que contraiga el VIH, al multiplicarlas por tres. Este rechazo les provoca, además, una baja autoestima y un mayor aislamiento. ${ }^{17,18}$

La idea del matrimonio homosexual por parte de los familiares demostró una actitud ridícula en su práctica, a pesar de los derechos constituidos por la Constitución Federal Mexicana (Reforma Mexicana 10 de junio 2011). A partir de esta ley, en México la familia puede considerarse como un fenómeno social y no un ideal a seguir, entendiéndose que la familia fomenta y protege conductas violentas hacia la homofobia, las cuales se aprenden como ideales de una sociedad. Es más probable que aquellos jóvenes homosexuales y transexuales que han sido apoyados por sus familias, formen una familia en el futuro, puesto que los resultados demuestran que el $90 \%$ de los jóvenes rechazados no quieren formarla. ${ }^{12,19-21}$

La sociedad mexicana y su cultura sexual están aún influenciadas por el conservadurismo de la heterosexualidad, y dicha norma sexual se perpetúa por medio del machismo y la homofobia. ${ }^{22,23}$ Una familia con fuerte vínculo emocional y compromiso familiar, producto del sentimiento de pertenencia, dará mejor acogida a este miembro con preferencia sexual homosexual que aquellas otras familias disfuncionales donde los vínculos y relaciones se desarrollen más por conveniencia o por funcionalidad. ${ }^{18,21-24}$

Las sociedades definen su alteridad, los límites del orden y la orientación de la conducta que resulta apropiada. Sin embargo, frente al orden instituido coexiste un orden del simulacro, soportado en lógica y actuaciones que se configuran al margen, desde la resistencia a ese orden pautado. ${ }^{25}$ ¿Cómo se puede explicar en el seno familiar que el homosexual sea parte de la sociedad cuando dicha cultura está llena de tabúes discriminatorios, que de alguna manera se perpetúan en la familia? Sus integrantes deben considerar que el individuo con diferente preferencia sexual es otro miembro más de la familia, con los mismos derechos y obligaciones, y que no puede ser delegado ni discriminado del seno familiar. La alteridad se cristaliza en formas inéditas de miedo y temor a todo aquello que es diferente, para el sujeto "desviado" de la normalidad. La sexualidad es un fenómeno social porque es histórico, cambiante y sólo definible en el contexto de una cultura; es, por ende, un constructo histórico. Como hecho sociocultural, la sexualidad contemporánea está fuertemente influida por el feminismo, la liberación sexual y la construcción de la identidad y por el orgullo gay y lésbico.

Este estudio tiene varias limitaciones como, por ejemplo, el de contar con una muestra muy limitada, lo que puede estar relacionado con la falta de reconocimiento social hacia la preferencia sexual diferente. Tampoco se consideraron determinantes sociales ni tipología familiar, aunque sí que se buscó dar inicio a dichos estudios dentro de instituciones de servicios de salud, ya que se trata de un grupo minoritario discriminado por estigmatizaciones sociales, familiares, culturales, económicas y de salud. 


\section{Conclusiones}

Es posible afirmar que el rechazo parental al hijo homosexual fue considerablemente alto en el grupo investigado. Cabe mencionar que estos pacientes deben ser abordados no sólo individualmente sino también en su entorno familiar. La búsqueda de alternativas, no sólo biomédicas, brindará la oportunidad de tener una aceptación de la expresión homosexual, disminuyendo la discriminación en el seno familiar y, por ende, en el institucional.

Por un lado, la construcción social en torno a la homosexualidad se instaura en el seno familiar ocasionando disfunción familiar y, por ende, rechazo familiar; este rechazo puede conducir a conductas de frustración, bordeando el aislamiento y la represión. La homosexualidad como hecho sociocultural ha incidido, en muy poco tiempo, en la transformación tanto de las pautas de afectividad como de cuidado de pareja, pero al mismo tiempo ha generado resistencia en ciertas estructuras sociales que le son adversas, tales como la iglesia, los partidos de derecha, etc.

La Medicina Familiar debe iniciar alternativas junto con la psicología y el trabajo social, para integrar dichas familias: valorando el grado de disfunción familiar asociado al rechazo familiar del paciente homosexual, y realizando terapias de intervención, con el objetivo de integrar a estos pacientes en su entorno familiar. La atención primaria de salud, debe fomentar la igualdad en los derechos universales de estos pacientes, así como su accesibilidad a los servicios de salud.

\section{Referencias}

1. Casas D, Jarillo E, Montealegre M, Irigoyen A, Casas I. La complejidad de la familia: una aproximación teórica desde la medicina familiar y la salud colectiva. Arch Med Fam. 2011;13(3):141-154.

2. Delval J. El desarrollo humano. México: Siglo veintiuno editores; 2009. p. 210-218.

3. Padilla S, Quintero M, Velázquez E. Genero y salud. México: Editorial Miguel Ángel Porrúa; 2012. p. 57-82.

4. Garrido L, Gil E. Estratégias familiares. Madrid: Alianza editorial; 1993. p. 181-183.

5. Fonseca C. Derechos Humanos, amor y sexualidad. México: Editorial Miguel Ángel Porrúa; 2013. p. 29-47.

6. Chavez J. Genero y familia. México: Editorial Plaza y Valdez; 2008. p. 11-20.

7. Barrón Rivera AJ, Palma Cham AG, Juárez Huerta L. Actitud del médico familiar ante el paciente con VIH/SIDA. Factores relacionados. Rev Med IMSS [Internet]. 2000 [última consulta el 2013 Jul 24];38(3):203-209. Disponible en: http://bases.bireme.br/cgi-bin/wxislind.exe/iah/ online/?IsisScript=iah/iah.xis\&src=google\&base=LILACS\&lang=p\&nextAction=Ink\&exprSearch=302861\&indexSearch=ID

8. Caitlin R, Huebner D, Diaz RM, Sanchez J. Family rejection as a predictor of negative health outcomes in white and Latino lesbian, gay, and bisexual young adults. Pediatrics [Internet]. 2009 [última consulta el 2013 Jul 18];123(1):346-352. Disponible en: http://dx.doi.org/10.1542/ peds.2007-3524

9. Diaz R, Ayala G, Bein E, Jenne J, Marin BV. The impact of homophobia, poverty, and racism on the mental health of Latino gay men. Am J Public Health. [Internet]. 2001 [última consulta el 2013 Jul 11];91(6):927-932. Disponible en: http://dx.doi.org/10.2105/AJPH.91.6.927

10. Binson D, Blair J, Huebner D, Woods J. Sampling in surveys of lesbian, gay, and bisexual people. In: Meyer IH, Northridge ME, editors. The Health of Sexual Minorities: Public Health Perspectives on Lesbian, Gay, Bisexual, and Transgender Populations. [Internet]. 2002 [última consulta el 2013 Jul 24];30(5):364-374. Disponible en: http://dx.doi.org/10.1016/S1054-139X(01)00415-3

11. Bontempo D, D'Augelli AR. Effects of at-school victimization and sexual orientation on lesbian, gay, or bisexual youths' health risk behavior. J Adolesc Health. [Internet]. 2002 [última consulta el 2013 Jul 24];30(5):364-374. Disponible en: http://dx.doi.org/10.1016/S1054-139X(01)00415-3

12. Ryan C, Futterman D. Lesbian and gay youth: Care and counseling. J Adolesc Med.1997;8(2):207-374.

13. Ardila R. Homosexualidad y psicología. Bogotá: Ed Manual Moderno; 1998. p. 75-8.

14. Foucault, M. Historia de la sexualidad (La voluntad de saber). México: Editorial Siglo Veintiuno Editores; 2004. p. 62-67.

15. Lozano V. El significado de homosexualidad en jóvenes de la Ciudad de México. Rev Enseñ Inv Psic. 2009; [Internet]. 2009 [última consulta el 2013 Jul 14];14(1):153-168. Disponible en: http://www.redalyc.org/articulo.oa?id=29214111

16. Verduzco L, Díaz-loving R. Medición de la homofobia en México: Desarrollo y validación. RIDEP. [Internet]. 2010 [última consulta el 2013 Jul 02];30(2):105-124. Disponible en: http://www.aidep.org/03_ridep/r30/RIDEP\%2030\%20-6.pdf

17. Granados J. La vivencia del rechazo en homosexuales universitarios de la Ciudad de México y situaciones de riesgo para VIH/sida. Salud Publica Mex. [Internet]. 2009 [última consulta el 2013 Jul 01];51(6):482-488. Disponible en: https://siid.insp.mx/textos/com-38121023.pdf 
18. Romero D. Homosexualidad y familia: ¿integración o rechazo? [tese na Internet]. Santigo de Chile: Universidad Academia de Humanismo Cristiano; 2011 [accesado 2013 Jul. 22]. Disponible en: http://bibliotecadigital.academia.cl/bitstream/123456789/214/1/Tesis\%20Final.pdf

19. Ilan H. Wilson P. Sampling Lesbian, gay and bisexual populations. J Couns Psychol. [Internet]. 2009 [última consulta el 2013 Jul 02];56(1):23-31. Disponible en: http://www.columbia.edu/ im15/papers/sampling.pdf

20. Hoffman N, Freeman K, Swann S. Healthcare preferences of lesbian, gay, bisexual, transgender and questioning youth. J Adolesc Health. [Internet]. 2009 [última consulta el 2013 Jul 15];45(3):222-229. Disponible en: http://www.jahonline.org/article/S1054-139X(09)00096-2/ abstract

21. Lock J, Steiner H. Gay, lesbian, and bisexual youth risks for emotional, physical, and social problems: results from a community-based survey. J Am Acad Child Adolesc [Internet]. 2009 [última consulta el 2013 Jul 15];45(3):222-229. Disponible en: http://www.jahonline.org/article/S1054139X(09)00096-2/abstract

22. Meyer IH. Prejudice, social stress, and mental health in lesbian, gay, and bisexual populations: conceptual issues and research evidence. Psychol Bull. [Internet]. 2003 [última consulta el 2013 Jun 30];129(5):674-697. Disponible en: http://dx.doi.org/10.1037/0033-2909.129.5.674

23. Lizarraga X. Una historia sociocultural de la homosexualidad: notas sobre un devenir silenciado. Barcelona: Editorial Paidós; 2003. p. 176-9.

24. Lamas M. Cuerpo: diferencia sexual y género. México: Editorial Taurus; 2002. p. 28-45.

25. Baudrillard J. Figuras de la alteridad. Madrid: Editorial Taurus; 2000. p. 34-45. 